\title{
Shoulder Manipulation After Distention Arthrography: Does Audible Cracking Affect Improvement in Adhesive Capsulitis? A Preliminary Study
}

\author{
Jun Kyu Choi, MD, Seok Beom Son, MD, Bum Jun Park, MD, \\ Seung Nam Yang, MD, PhD, Joon Shik Yoon, MD, PhD
}

Department of Physical Medicine and Rehabilitation, Korea University Guro Hospital, Seoul, Korea

Objective To investigate whether an audible cracking sound during shoulder manipulation following distention arthrography is clinically significant in patients with adhesive capsulitis of the shoulder.

Methods A total of 48 patients ( 31 women, 17 men) with primary adhesive capsulitis of the shoulder completed the study. All participants underwent C-arm-guided arthrographic distention of the glenohumeral joint with injections of a corticosteroid and normal saline. After distention, we performed flexion and abduction manipulation of the shoulder. The patients were grouped into sound and non-sound groups based on the presence or absence, respectively, of an audible cracking sound during manipulation. We assessed shoulder pain and disability based on a Numeric Rating Scale (NRS), the Shoulder Pain and Disability Index (SPADI), and passive range of motion (ROM) measurements (flexion, abduction, internal and external rotation) before the procedure and again at 3 weeks and at 6 weeks after the intervention.

Results The patients were divided into two groups: 21 were included in the sound group and 27 in the nonsound group. In both groups, the results of the NRS, SPADI, and ROM assessments showed statistically significant improvements at both 3 and 6 weeks after the procedure. However, there were no significant differences between the two groups except with respect to external rotation at 6 weeks, at which time the sound group showed a significant improvement in external rotation when compared with the non-sound group ( $<<0.05)$.

Conclusion These findings showed that manipulation following distention arthrography was effective in decreasing pain and increasing shoulder range of motion. In addition, the presence of an audible cracking sound during manipulation, especially on external rotation, was associated with better shoulder range of motion.

Keywords Adhesive capsulitis, Distention arthrography, Manipulation, Cracking sound, Shoulder range of motion

Received April 20, 2015; Accepted June 4, 2015

Corresponding author: Joon Shik Yoon

Department of Physical Medicine and Rehabilitation, Korea University Guro Hospital, 148 Gurodong-ro, Guro-gu, Seoul 08308, Korea Tel: +82-2-2626-1500, Fax: +82-2-2626-1513, E-mail: rehab46@korea.ac.kr

() This is an open-access article distributed under the terms of the Creative Commons Attribution Non-Commercial License (http://creativecommons.org/ licenses/by-nc/4.0) which permits unrestricted noncommercial use, distribution, and reproduction in any medium, provided the original work is properly cited. Copyright $\odot 2015$ by Korean Academy of Rehabilitation Medicine 


\section{INTRODUCTION}

Adhesive capsulitis, known as frozen shoulder or painful stiff shoulder, is a common cause of shoulder pain that affects $2 \%$ to $5 \%$ of the general population [1]. The pain and impaired mobility can lead to progressive underutilization of the affected shoulder and ultimately the loss of function $[1,2]$. The treatment objectives in adhesive capsulitis are to relieve pain, regain motion of the shoulder, and restore function. In patients with severe pain or a limited range of motion (ROM), physiotherapy, exercise, and oral medication are recommended. Other approaches include intra-articular injections, capsular distention, manipulation under anesthesia, and surgery. In a study comparing manipulation and intra-articular injection, there was no difference in the outcomes [3]. No single, standard treatment is universally accepted in the management of adhesive capsulitis. Combination treatment such as distention arthrography with manipulation using local anesthesia is also a safe and effective way to address frozen shoulder [4].

During manipulation, one can hear a typical cracking sound known as characteristic crepitus. This sound is believed to be reflect the loosening and tearing of the adhesive components of the shoulder joint $[5,6]$. Several studies have reported that shoulder mobility increased after this audible cracking sound $[7,8]$. Whereas some researchers have reported that a cracking sound was audible in all cases upon manipulation of the shoulders in patients with adhesive capsulitis $[6,8]$, others reported hearing a cracking sound in only some cases $[5,9]$. To our knowledge, the clinical significance of this cracking sound during manipulation has not yet been addressed.

We aimed to investigate whether an audible cracking sound during shoulder manipulation has clinical significance in patients with adhesive capsulitis of the shoulder. We hypothesized that the presence of an audible cracking sound would indicate a positive clinical outcome.

\section{MATERIALS AND METHODS}

\section{Subjects}

A total of 54 consecutive patients who were diagnosed with adhesive capsulitis were enlisted from the Department of Physical Medicine and Rehabilitation in Korea University Guro Hospital in Seoul, Korea, from May 1,
2013, to April 1, 2014. Every participant provided informed consent after obtaining approval from the regional Institutional Review Board of the Korea University Guro Hospital.

The subjects in this study were diagnosed with adhesive capsulitis on the basis of their medical history and physical examination. Inclusion criteria were age 18 years or older, shoulder pain for more than 3 months, and restriction of passive motion of $30^{\circ}$ in at least two planes of movement when compared with the opposite side [10]. Subjects were excluded if they had a painful shoulder following severe trauma, a previous stroke with motor deficits, or a known allergy to a local anesthetic agent, iodine, or steroids.

A plain radiograph and ultrasonography were obtained in each case, and other disorders were excluded, such as fracture, shoulder dislocation, significant glenohumeral arthritis, rotator cuff rupture, and calcific tendinopathy. Sonographic evaluations were performed by one physiatrist (J. S. Yoon) with 15 years of experience in musculoskeletal sonography.

\section{Clinical evaluations}

We measured the intensity of shoulder pain by means of a Numeric Rating Scale (NRS), the Shoulder Pain and Disability Index (SPADI), and passive ROM testing at baseline and at 3 weeks and 6 weeks after the patients underwent distention arthrography. The SPADI is a selfadministered and consists of 13 items divided into two subscales: pain (5 items) and disability (8 items) [11]. The SPADI is scored on a scale of 0 to 100 by averaging the individual scores from the two subscales, and the highest scores indicate the most severe pain and disability.

Shoulder passive ROM was measured in four different directions of movement: forward flexion/abduction from neutral and external/internal rotation from a $45^{\circ}$ abduction. A physiotherapist assisted the patient in achieving maximum movement and measured the extent of passive ROM in all four directions. All ROMs were measured in a supine position.

The NRS and SPADI were evaluated by a physician (B. J. Park) who was blinded to the patient's history, results of the previous physical examination, and the NRS and SPADI scores. Passive ROMs were assessed by a physiotherapist who was blinded to the results of previous ROM tests. 


\section{Distention arthrography}

All participants underwent fluoroscopically guided arthrographic distention of the glenohumeral joint. All injections were performed by the same clinician who performed the sonographic evaluations. Procedures were carried out under strict aseptic conditions. With the patient in the supine position, a local anesthetic agent $(2 \%$ lidocaine hydrochloride) was infiltrated into the skin and subcutaneous soft tissue. A 22-gauge, 3.5-inch spinal needle (Spinocan; B. Braun Melsungen AG, Melsungen, Germany) was inserted anteriorly into the glenohumeral joint under fluoroscopic guidance, and approximately 3 $\mathrm{mL}$ of non-ionic contrast substance (Xenetix; Guerbet, Rome, Italy) was injected. The position of the needle was confirmed by fluoroscopy, and the integrity of the intraarticular structures was verified. This was followed by an injection consisting of $40 \mathrm{mg}$ of triamcinolone (in $1 \mathrm{~mL}$ ), $9 \mathrm{~mL}$ of $0.5 \%$ lignocaine, and $10 \mathrm{~mL}$ of normal saline. This injection ( $20 \mathrm{~mL}$ total) was given to all the patients to distend the glenohumeral joint [12].

\section{Manipulation}

All shoulder manipulations were performed by the same clinician who carried out the distention arthrography procedure with 10 years of experience performing shoulder manipulation. Manipulation was then initiated by holding the patient's humerus and manipulating it initially into full abduction, then flexion with stabilization of the scapula. After achieving the maximum possible ROM, the arm position was maintained for 30 to 60 seconds in alternately full abduction and flexion. This sequence was repeated until the maximum possible ROM was achieved. During manipulation, any typical audible cracking sound was recorded by a physician (J. K. Choi) who was blinded to the patient's history and he results of the previous physical examination. Based on the presence or absence of an audible cracking sound, the patients were classified into sound and non-sound groups, respectively. After manipulation, we recommended that the participants perform only forward stretching exercises to increase ROM.

\section{Adverse effects}

To determine whether there were any complications of the intervention, we arranged to follow up with an examination for symptoms and a careful inspection of the shoulder immediately after the procedure and again at 3 weeks. In addition, subjects were asked to report any adverse effects of the intervention, as elicited by openended questions.

\section{Statistical analysis}

Data were analyzed using SPSS ver. 20.0 software (IBM, Armonk, NY, USA). A repeated-measures analysis of variance (ANOVA) was used to compare the degree of improvement between the two groups in terms of the NRS, ROM of flexion, abduction, external and internal rotation of the shoulder, and SPADI at each time point; this was followed by a Bonferroni post hoc analysis. A stepwise multivariate linear regression analysis was also used to explore the effects of covariates (age, gender, disease duration, audible cracking sound) on improvements in NRS, SPADI, and each type of ROM. Independent t-tests and chi-square tests were used to compare baseline characteristics, such as age, sex, side affected, and disease duration, between the two groups. All tests of statistical significant were performed at the $5 \%$ level of significance.

\section{RESULTS}

From the initial 68 patients who were screened, 54 were recruited after 15 patients who did not meet the inclusion criteria were excluded. Of these 54 patients, 23 (42.59\%) patients had an audible cracking sound during ma-

Table 1. General characteristics of the patients

\begin{tabular}{lccc}
\hline \multicolumn{1}{c}{ Characteristic } & Sound group $(\mathbf{n = 2 1})$ & Non-sound group $(\mathbf{n}=\mathbf{2 7})$ & p-value \\
\hline Age (yr) & $54.10 \pm 7.54$ & $57.78 \pm 9.98$ & 0.166 \\
Sex (male:female) & $8: 13$ & $9: 18$ & 0.732 \\
Duration of symptoms (mo) & $8.90 \pm 3.76$ & $12.44 \pm 8.84$ & 0.104 \\
Affected side (right:left) & $14: 7$ & $13: 14$ & 0.199 \\
\hline
\end{tabular}

Values are presented as mean \pm standard deviation or number. 
nipulation. After the procedure, 2 patients in the sound group and 4 patients in the non-sound group were lost to follow-up. Therefore, the sound group consisted of 21 patients (mean age, $54.10 \pm 7.54$ years; 8 men, 13 women), and the non-sound group consisted of 27 patients (mean age, $57.78 \pm 9.98$ years; 9 men, 18 women). General characteristics of these 48 patients are presented in Table 1. There were no significant differences in age, sex, disease duration, or affected side between the sound group and the non-sound group.

\section{Treatment effect between the groups}

The NRS significantly decreased at 3 and 6 weeks after the procedure in both groups $(\mathrm{p}<0.05)$ (Table 2, Fig. 1). However, no significant differences were observed

Table 2. Comparison of NRS and SPADI after intervention

\begin{tabular}{lccc}
\hline & Baseline & After 3 wk & After 6 wk \\
\hline NRS & & & \\
$\quad$ Sound & $6.6 \pm 2.3$ & $3.6 \pm 2.4^{*}$ & $2.6 \pm 2.0^{*}$ \\
$\quad$ Non-sound & $6.9 \pm 2.6$ & $4.3 \pm 2.5^{*}$ & $3.4 \pm 2.1^{*}$ \\
SPADI & & & \\
$\quad$ Sound & $63.1 \pm 31.3$ & $28.4 \pm 21.2^{*}$ & $18.8 \pm 14.7^{*}$ \\
$\quad$ Non-sound & $64.2 \pm 30.3$ & $37.7 \pm 26.6^{*}$ & $28.4 \pm 24.3^{*}$ \\
\hline
\end{tabular}

Values are presented as means \pm standard deviation. NRS, Numeric Rating Scale; SPADI, Shoulder Pain and Disability Index.

${ }^{*} \mathrm{p}<0.05$.

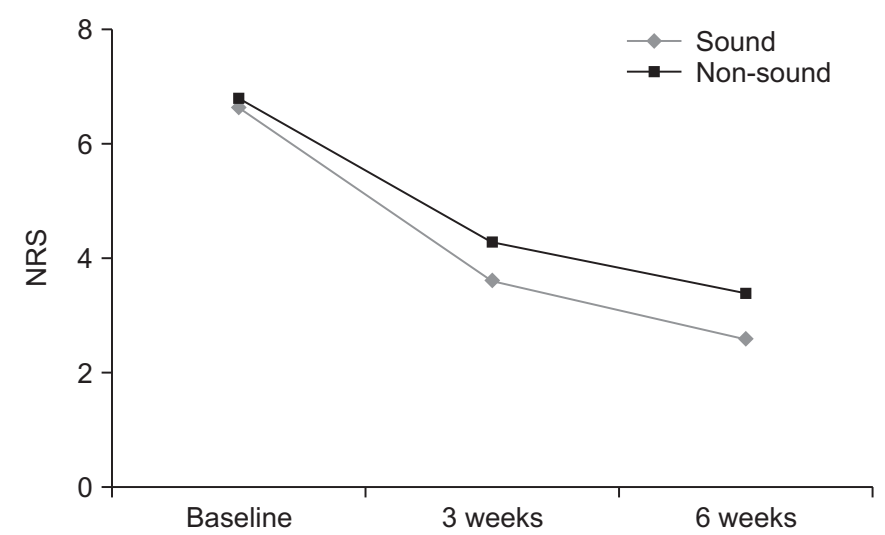

Fig. 1. Comparison of changes in the Numeric Rating Scale (NRS) in the sound group and the non-sound group. The NRS significantly improved at 3 and 6 weeks in both groups. However, no significant differences are observed between the groups in the NRS at baseline and at 3 and 6 weeks. between the groups in the NRS at baseline or at 3 and 6 weeks after the procedure. The SPADI scores also significantly improved in both groups at 3 and 6 weeks after the procedure (Fig. 2). However, no significant differences were observed between the groups in the SPADI scores at

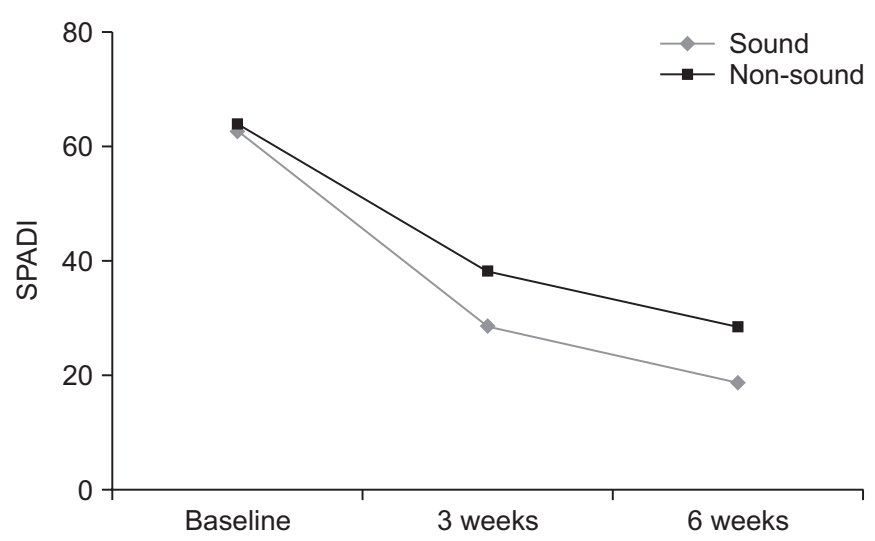

Fig. 2. Comparison of changes in the Shoulder Pain and Disability Index (SPADI) in the sound group and the nonsound group. The SPADI significantly improved at 3 and 6 weeks in both groups. However, no significant differences are observed between the groups in the SPADI at baseline and at 3 and 6 weeks.

Table 3. Comparison of shoulder ROM after intervention

\begin{tabular}{|c|c|c|c|}
\hline & Baseline & After 3 wk & After 6 wk \\
\hline \multicolumn{4}{|l|}{ Flexion } \\
\hline Sound & $139.3 \pm 23.7$ & $157.4 \pm 15.9^{*}$ & $161.0 \pm 13.0^{*}$ \\
\hline Non-sound & $140.6 \pm 18.8$ & $156.9 \pm 15.9^{*}$ & $163.7 \pm 13.3^{*}$ \\
\hline \multicolumn{4}{|l|}{ Abduction } \\
\hline Sound & $118.6 \pm 32.2$ & $160.24 \pm 26.1^{*}$ & $165.48 \pm 19.4^{*}$ \\
\hline Non-sound & $122.6 \pm 40.9$ & $152.2 \pm 27.6^{*}$ & $154.63 \pm 28.2^{*}$ \\
\hline \multicolumn{4}{|c|}{ External rotation } \\
\hline Sound & $52.4 \pm 27.5$ & $68.1 \pm 19.5^{*}$ & $78.5 \pm 17.7^{\text {*a }}$ \\
\hline Non-sound & $56.5 \pm 26.3$ & $63.3 \pm 20.3^{*}$ & $67.0 \pm 18.0^{*}$ \\
\hline \multicolumn{4}{|c|}{ Internal rotation } \\
\hline Sound & $36.9 \pm 19.9$ & $50.5 \pm 22.6^{*}$ & $54.1 \pm 21.5^{*}$ \\
\hline Non-sound & $44.3 \pm 21.9$ & $51.1 \pm 18.5^{*}$ & $58.2 \pm 16.8^{*}$ \\
\hline \multicolumn{4}{|l|}{ Total ROM } \\
\hline Sound & $347.2 \pm 94.9$ & $436.2 \pm 66.8^{*}$ & $461.4 \pm 53.4^{*}$ \\
\hline Non-sound & $363.9 \pm 93.8$ & $423.5 \pm 69.0^{*}$ & $443.5 \pm 59.7^{*}$ \\
\hline \multicolumn{4}{|c|}{$\begin{array}{l}\text { Values are presented as means } \pm \text { standard deviation. } \\
\text { ROM, range of motion. } \\
\text { a) Statistically significant difference between the two pa } \\
\text { tient groups. } \\
{ }^{*} \mathrm{p}<0.05 \text {. }\end{array}$} \\
\hline
\end{tabular}




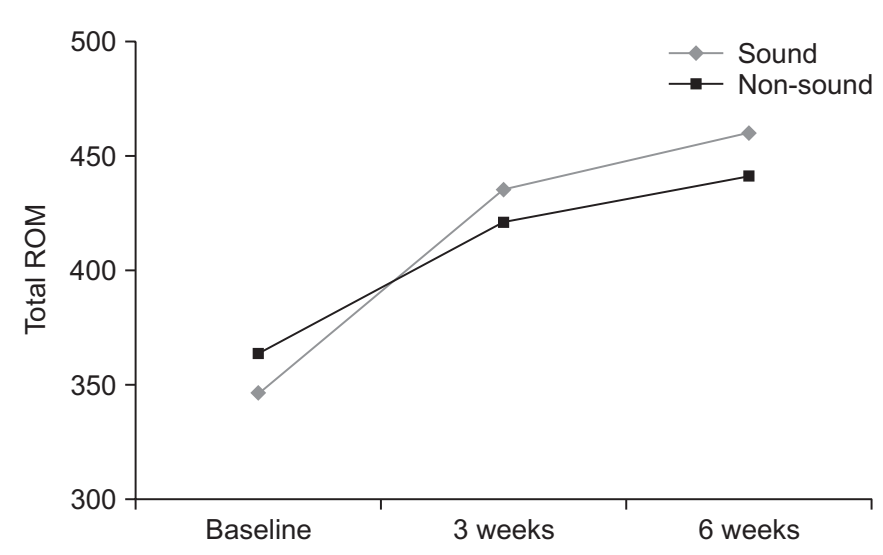

Fig. 3. Comparison of changes in total range of motion (ROM) in the sound group and the non-sound group. The total ROM significantly improved at 3 and 6 weeks in both groups. However, no significant differences are observed between the groups in the total ROM at baseline and at 3 and 6 weeks.

baseline or at 3 and 6 weeks after the procedure.

With respect to changes in passive ROM, there were significant improvements in both groups in shoulder abduction, flexion, external rotation, and internal rotation at 3 and 6 weeks after treatment (Table 3, Fig. 3). According to a repeated-measures ANOVA, the sound group showed a significant improvement in shoulder external rotation at 6 weeks, as compared with the non-sound group $(\mathrm{p}<0.05)$ (Fig. 4); the results in the sound group and nonsound group, respectively, were $52.4 \pm 27.5$ and $56.5 \pm 26.3$ at baseline and $78.5 \pm 17.7$ and $67.0 \pm 18.0$ at 6 weeks. By regression analysis, an audible cracking sound was shown to be the only significant factor associated with improved external rotation at 6 weeks $\left(R^{2}=0.101, \beta=0.301, p=0.037\right)$. Patients' age, gender, and disease duration were not included as significant factors on regression analysis for other outcome improvements.

No serious complications such as fracture, neurologic complications, infection, allergic reaction, nausea, or dizziness were observed during the study period.

\section{DISCUSSION}

Our results indicate that distention arthrography with manipulation in patients with adhesive capsulitis is effective in improving function, reducing pain, and increasing ROM at 3 and 6 weeks. Furthermore, an audible cracking sound during manipulation suggested that improvement in external rotation at 6 weeks was greater than if

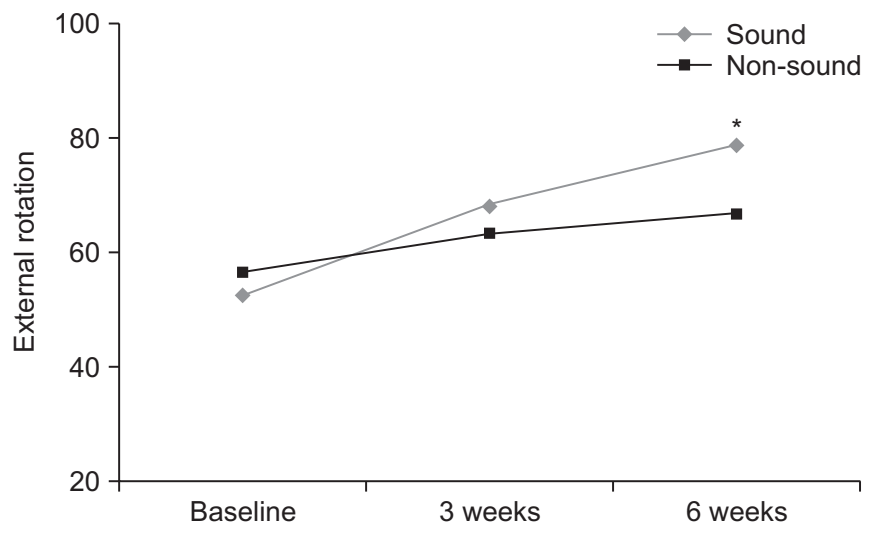

Fig. 4. Comparison of changes in external rotation in the sound group and the non-sound group. The external rotation significantly improved at 3 and 6 weeks in both groups. As compared with the non-sound group, the sound group shows a significant improvement from baseline in shoulder external rotation at 6 weeks $\left({ }^{*} \mathrm{p}<0.05\right)$.

no cracking sound was heard. An audible cracking sound was heard in 23 ( $42.59 \%$ ) of the original 54 patients in our study and is believed to be the result of loosening and tearing of the adhesive components of the joint $[5,6]$. If this sound is heard during manipulation, it means that the adhesive component of the shoulder is torn $[6,13]$. In our study, the presence of a cracking sound was not directly associated with pain relief but was associated with a wider range of external rotation.

The joint space capacity in the normal shoulder joint is greater than $16 \mathrm{~mL}$, whereas in adhesive capsulitis it is less than $10 \mathrm{~mL}$ [7]. Mao et al. [14] investigated the correlation between the difference in shoulder joint space capacity and joint ROM improvement after physical therapy in patients with adhesive capsulitis. In their study, the entire shoulder ROM, including flexion, abduction, external rotation, and internal rotation, was significantly improved after conservative treatment that included physical modalities and exercise. Moreover, the joint space capacity as measured on shoulder arthrography increased significantly after such treatment; however, this increase was significantly correlated with improvement only in external rotation and not in flexion, abduction, or internal rotation.

In our study, ROM, which included all four of these directions, improved in both the sound and non-sound groups after distention arthrography and manipulation. During manipulation, an audible cracking sound was 
heard mostly during flexion, yet a significant difference in improvement between the two groups was observed only for external rotation. One explanation for this finding might be an increased joint space in the sound group. The restriction of passive motion in multiple planes, particularly on external rotation, is a significant finding in adhesive capsulitis [15]. Mao et al. [14] discovered that increased joint space correlated more with external rotation than with any other type of ROM. Based on their results; we hypothesize that if the shoulder adhesion is torn properly, the joint space would increase and external rotation would be the most likely ROM to improve.

Some studies have reported that the cracking sound is heard in all manipulated shoulders [6,8], but other studies have reported that the cracking sound is heard in only some cases $[5,9]$. This discrepancy is probably due to clinical differences-that is, if the cracking sound is not loud enough to be heard, it can be missed.

Arthrographic distention can help reduce pain and increase ROM in patients with adhesive capsulitis $[5,7,11]$. The effects and benefits of manipulation in this disorder have been well documented in several published reports $[3,8]$. Combination treatment using arthrographic distention and manipulation under general anesthesia, as described by Ekelund and Rydell [16], led to a rapid decrease in pain and an improvement in ROM at 4 to 6 weeks.

A combination of arthrographic distention and manipulation under local anesthesia was also described by van Royen and Pavlov [4]. They studied 22 patients and showed that distention along with manipulation without general anesthesia could provide sufficient gains in ROM. After the intervention, shoulder ROM improved, and the mean increases in range at 12 weeks were $86.8^{\circ}$ for abduction, $71.5^{\circ}$ for forward flexion, $38.8^{\circ}$ for internal rotation, and $48.9^{\circ}$ for external rotation. These authors also concluded that distention and manipulation under local anesthesia is a safe and effective method for treating adhesive capsulitis. Similar to the results reported in previous studies, we also observed overall improvement in the ROM in every plane at 6 weeks after the procedure. The mean increases in range for all 48 patients were $38.5^{\circ}$ for abduction, $22.4^{\circ}$ for forward flexion, $16.3^{\circ}$ for internal rotation, and $15.4^{\circ}$ for external rotation. In addition to ROM, we also evaluated NRS and SPADI to measure pain and disability related to adhesive capsulitis. After the procedure, we noted significant improvements in function and ROM and a reduction in pain.

Some important limitations to this study should be considered. First, the number of patients was small and the follow-up period was short. A larger number of patients with a longer follow-up period will be needed to confirm the clinical effect of the cracking sound during manipulation. Second, if the cracking sound is not loud enough, it could be missed. Third, although we performed combination treatment-an intra-articular steroid injection, distention arthrography, and manipulation-in both the sound group and the non-sound group, it is possible that the addition of the injection and distention arthrography to manipulation could have affected the outcomes. Because our results are confined to the effect of an audible cracking sound during manipulation after distention arthrography, further study that involves manipulation alone is needed. Fourth, although in most cases the cracking sound was heard on shoulder flexion during manipulation, it was difficult to determine exactly when the sound occurred during the two types of movement, flexion or abduction. Additional studies to correlate the specific movement and improvement in each type of ROM are needed.

In conclusion, our results showed that distention arthrography along with manipulation led to a reduction in pain and improvements in both ROM and general shoulder function in patients with adhesive capsulitis. The audible cracking sound during manipulation did not seem to be associated with pain relief, but it did result in a better range of shoulder external rotation at 6 weeks after the intervention.

\section{CONFLICT OF INTEREST}

No potential conflict of interest relevant to this article was reported.

\section{REFERENCES}

1. Carette S. Adhesive capsulitis: research advances frozen in time? J Rheumatol 2000;27:1329-31.

2. Rizk TE, Gavant ML, Pinals RS. Treatment of adhesive capsulitis (frozen shoulder) with arthrographic capsular distension and rupture. Arch Phys Med Rehabil 1994;75:803-7. 
3. Jacobs LG, Smith MG, Khan SA, Smith K, Joshi M. Manipulation or intra-articular steroids in the management of adhesive capsulitis of the shoulder? A prospective randomized trial. J Shoulder Elbow Surg 2009;18:348-53.

4. van Royen BJ, Pavlov PW. Treatment of frozen shoulder by distension and manipulation under local anaesthesia. Int Orthop 1996;20:207-10.

5. Amoretti N, Grimaud A, Brocq O, Roux C, Dausse F, Fournol M, et al. Shoulder distension arthrography in adhesive capsulitis. Clin Imaging 2006;30:254-6.

6. Loew M, Heichel TO, Lehner B. Intraarticular lesions in primary frozen shoulder after manipulation under general anesthesia. J Shoulder Elbow Surg 2005;14:1621.

7. Lundberg BJ. The frozen shoulder: clinical and radiographical observations. Acta Orthop Scand Suppl 1969;119:1-59.

8. Kivimaki J, Pohjolainen T, Malmivaara A, Kannisto M, Guillaume J, Seitsalo S, et al. Manipulation under anesthesia with home exercises versus home exercises alone in the treatment of frozen shoulder: a randomized, controlled trial with 125 patients. J Shoulder Elbow Surg 2007;16:722-6.

9. Khan JA, Devkota P, Acharya BM, Pradhan NM, Shreshtha SK, Singh M, et al. Manipulation under local anesthesia in idiopathic frozen shoulder: a new effective and simple technique. Nepal Med Coll J 2009;
11:247-53.

10. Buchbinder R, Youd JM, Green S, Stein A, Forbes A, Harris A, et al. Efficacy and cost-effectiveness of physiotherapy following glenohumeral joint distension for adhesive capsulitis: a randomized trial. Arthritis Rheum 2007;57:1027-37.

11. Roach KE, Budiman-Mak E, Songsiridej N, Lertratanakul Y. Development of a shoulder pain and disability index. Arthritis Care Res 1991;4:143-9.

12. Tveita EK, Tariq R, Sesseng S, Juel NG, Bautz-Holter E. Hydrodilatation, corticosteroids and adhesive capsulitis: a randomized controlled trial. BMC Musculoskelet Disord 2008;9:53.

13. Goldberg BA, Scarlat MM, Harryman DT 2nd. Management of the stiff shoulder. J Orthop Sci 1999;4:46271.

14. Mao CY, Jaw WC, Cheng HC. Frozen shoulder: correlation between the response to physical therapy and follow-up shoulder arthrography. Arch Phys Med Rehabil 1997;78:857-9.

15. Kelley MJ, Shaffer MA, Kuhn JE, Michener LA, Seitz AL, Uhl TL, et al. Shoulder pain and mobility deficits: adhesive capsulitis. J Orthop Sports Phys Ther 2013; 43:A1-31.

16. Ekelund AL, Rydell N. Combination treatment for adhesive capsulitis of the shoulder. Clin Orthop Relat Res 1992;(282):105-9. 